\title{
Evaluating a Programme for Intercultural Competence in Psychotherapist Training: A Pilot Study
}

\author{
Ulrike von Lersner $^{\mathrm{a}}$, Kirsten Baschin ${ }^{\mathrm{a}}$, Nora Hauptmann ${ }^{\mathrm{a}}$ \\ [a] Department of Psychology, Humboldt University of Berlin, Berlin, Germany.
}

Clinical Psychology in Europe, 2019, Vol. 1(3), Article e29159, https://doi.org/10.32872/cpe.v1i3.29159

Received: 2018-08-21 • Accepted: 2019-06-07 • Published (VoR): 2019-09-20

Handling Editor: Cornelia Weise, Philipps-University of Marburg, Marburg, Germany

Corresponding Author: Ulrike von Lersner, Department of Psychology, Humboldt-Universität zu Berlin, Rudower Chaussee 18, 12489 Berlin, Germany. E-mail: ulrike.von.lersner@hu-berlin.de

\section{Abstract}

Background: Great cultural diversity among clients poses considerable challenges to mental health service providers. Therefore, staff in the mental health sector needs to be adequately trained. To date, however, there is little empirical evidence regarding such training. The present pilot study evaluates the effect of a standardised training programme to improve the intercultural competence of therapists.

Method: Intercultural competence and therapeutic relationship were measured three times (pre, post and follow-up) in $\mathrm{N}=29$ psychotherapists. A control group of $\mathrm{N}=48$ therapists was included at pre-test to control for covariables.

Results: The data show a significant increase in intercultural competence as well as an improvement in the therapeutic relationship. Interestingly, this positive outcome extends to nonimmigrant clients.

Conclusion: The results confirm the assumption that culture is not limited to ethnic or national background but includes other dimensions such as age, gender and socioeconomic status which shape illness beliefs and expectations in the psychotherapeutic context. Therefore, intercultural competence can be considered a general therapeutic skill that can be taught in short interventions like the one developed in this study.

\section{Keywords}

evaluation, intercultural competence, diversity, psychotherapy, migration, awareness 


\section{Highlights}

- Mental health services and practitioners need to be able to respond appropriately to increasing cultural diversity.

- Intercultural competence in psychotherapy can be enhanced by special training programmes.

- These training programmes should focus on three components: intercultural knowledge, cultural awareness and culture-specific therapeutic skills.

As a consequence of continuously increasing global mobility, as well as war and environmental migration, cultural diversity in Western societies is growing rapidly. These processes are generating a degree of cultural diversity that requires mental health service administrators and practitioners to be able to respond appropriately.

Yet, in mental health services a considerably smaller percentage of immigrants is being treated now than would be expected considering their overall population share. This has been explained by some as a product of lower service use by clients with a migration background (Chen \& Rizzo, 2010; Claassen, Ascoli, Berhe, \& Priebe, 2005; Koch, Hartkamp, Siefen, \& Schouler-Ocak, 2008; Lindert et al., 2008; Machleidt, Behrens, Ziegenbein, \& Calliess, 2007; Ta et al., 2015). Previous studies have also shown that institutional barriers can hamper service uptake, including problems of language and other means of communication (Claassen et al., 2005; Kirmayer et al., 2011; Yeo, 2004), perceived or expected discrimination, and structural and financial barriers (Chen \& Rizzo, 2010; Kirmayer et al., 2011). Moreover, surveys of psychotherapists have shown a high degree of insecurity and helplessness in intercultural contexts, which can lead to a greater likelihood of rejecting them as patients or to higher dropout rates (de Haan, Boon, de Jong, \& Vermeiren, 2018; von Lersner, 2015; Wohlfart, Hodzic, \& Özbek, 2006).

The status quo, outlined above, suggests that an intercultural opening up of the mental health sector is urgently needed (Kirmayer et al., 2011; Machleidt et al., 2007), implying an adaptation of institutional and organisational structures to the needs of immigrant clients. Meanwhile, Benish, Quintana, and Wampold (2011) in their meta-analysis have suggested that intercultural therapies have better outcomes when therapists include patients' culturally shaped explanatory models in treatment. Furthermore, therapists should be better prepared for this group of clients through their professional training, in order to reduce insecurities and improve treatment outcomes in the long run (Aggarwal, Cedeño, Guarnaccia, Kleinman, \& Lewis-Fernández, 2016; Kirmayer, 2012; von Lersner, Baschin, Wormeck, \& Mösko, 2016). 


\section{Intercultural Competence Training}

Even though intercultural competence appears to be of high relevance in clinical settings, evaluations of intercultural training in the psychotherapeutic context are unfortunately still very rare (Kulik \& Roberson, 2008; Mösko, Baschin, Längst, \& von Lersner, 2012). To our knowledge, there has been no such training evaluation in German-speaking areas.

In the present study, we evaluated an intercultural training programme for therapists aimed at improving intercultural competence and therapeutic relationship. Thus, we evaluated cognitive, skill-based and affective learning, as well as possible improvements in the therapeutic relationship, as components of behavioural change that should emerge after training. Since the training programme was being conducted for the first time, our evaluation has the character of a pilot study.

Before discussing the intercultural training, the concept of culture as it is used in the present study needs to be defined. According to Geertz (1973, p. 83), culture describes 'a system of inherited conceptions expressed in symbolic forms by means of which men communicate, perpetuate, and develop their knowledge about, and attitudes toward, life'. Thus, it can be said to refer to a set of values and norms shared by a group of people independently of their national or ethnic backgrounds.

Meanwhile, in its current usage, the term intercultural competence stands for a wide range of definitions associated with numerous practical implications (Steinhäuser, Martin, von Lersner, \& Auckenthaler, 2014). However, a widely used concept in clinical settings is the one formulated by Sue and Sue (1990), which includes the following three dimensions:

- Awareness: Exploration of and reflection on one's own cultural embeddedness as well as its influence on perceptions of clients and the formation of the therapeutic relationship.

- Knowledge: Knowledge of the cultural background of the client and possible implications for his or her worldview.

- Skills: The development of culturally sensitive intervention strategies and techniques.

These three components, which can be said to constitute the basis of effective treatment in intercultural settings, represent a goal that is unlikely to be achieved in a one-off training session; rather, it requires an active, ongoing process that practitioners have to go through over a longer period of time (Guzder \& Rousseau, 2013).

The model developed by Sue and Sue (1990) has also become the basis of national guidelines on intercultural competence in various countries and organisations, such as the Multicultural Guidelines of the American Psychological Association (2003, 2008) and the Guidelines for Training in Cultural Psychiatry (Kirmayer et al., 2012). They have also been applied in a European strategy paper on intercultural competence in the mental health sector (Bennegadi, 2009) and in the German Guidelines for the Training of Intercultural Competence of Psychotherapists (von Lersner et al., 2016). 
Intercultural competence training can be carried out in multiple ways and can be quite heterogeneous with regard to target groups, duration, methods used and content, to name only a few dimensions. The majority of intercultural training evaluation studies in the health sector have been conducted with nursing staff and doctors as well as university students as subjects (Delgado et al., 2013; Khanna, Cheyney, \& Engle, 2009).

Four systematic reviews, covering 69 studies from 1980 through to 2010, have analysed the effectiveness of intercultural training for nursing staff and medical doctors mainly in the US (Beach et al., 2005; Chipps, Simpson, \& Brysiewicz, 2008; Lie, Lee-Rey, Gomez, Bereknyei, \& Braddock, 2011; Price et al., 2005). Beach et al. (2005) reported very good evidence of increased cultural knowledge among doctors and nursing staff as a result of such training, a result that was replicated by Chipps et al.'s (2008) study focusing on staff in rehabilitation centres. There is also good evidence from these studies that awareness and skills (see Sue \& Sue, 1990) can change and improve through such training. An evaluation of training programmes by Kulik and Roberson (2008) also reported large benefits in intercultural awareness and knowledge across target groups and training settings, but little improvements on the skills dimension. Regarding the quality of training schemes, both Lie et al. (2011) and Price and colleagues (2005) suggest that the quality of the evaluation studies examined was only low to moderate: most of them failed to control for confounding variables and effect sizes varied between zero and moderate. Thus, in contrast to the large number of training schemes available, there is a conspicuous lack of transparently documented and published studies on their effectiveness.

One concept that is widely used in intercultural competence training-and in the training programme evaluated in our study too-is the diversity approach. According to this perspective, there are six diversity dimensions that can influence perceptions of commonality and difference and are likely to lead to forms of discrimination: age; gender; sociocultural background, including migration history and skin colour; handicaps and skills; sexual orientation; and religion (van Keuk, Ghaderi, Joksimovic, \& David, 2011). Diversity training can focus on one or more of these dimensions, implying that cultural background is only one possible dimension. Participants should learn to be aware of the different types of diversity among their clients and how to deal with them in a positive way. A meta-analysis of the effectiveness of diversity training by Kalinoski et al. (2013) found significant effects on knowledge and skills but none on attitudes and awareness. Their analysis of training methods and structural factors suggested that training sessions lasting 12-16 hours and applying active training methods such as role play, discussion or critical incident technique had the greatest effectiveness, with training spread over multiple sessions being more effective than one-session programmes.

Effective training should have a positive impact on the cultural competence of the therapist as well as improving the therapeutic process, wherein the therapeutic relationship is of special interest. Norcross (2010, p. 113) defines the client-therapist relationship as 'the feelings and attitudes that therapist and client have toward one another and how 
these are expressed'. Meanwhile, Khanna and colleagues (2009) describe the therapeutic relationship within the intercultural therapeutic process as an important predictor of compliance and outcomes. Therefore, the present pilot study focuses on this aspect as well. With regard to efficiency, it is also relevant to evaluate whether rather short interventions, such as the one developed for the present study, can initiate such positive change.

From the start, we assumed that our programme would have a positive impact on the intercultural competence of participants, and that these effects would be quantifiable across our three points of measurement-before, immediately after and three months after participation-of the programme. We further hypothesised that having to consider intercultural issues during the programme would affect the therapeutic relationship in a beneficial way. As such an impact would probably only unfold with time (Guzder \& Rousseau, 2013; Kulik \& Roberson, 2008), we assumed that the effects would be most observable during the follow-up analysis. Further, as another goal of the pilot study was to assess whether the training programme could serve groups of therapists with different levels of experience, we included therapists in training as well as experienced therapists and analysed the effects of the training on them separately. We assumed that participants would benefit from the training regardless of their level of therapeutic experience; that is, that therapists in training would benefit from the programme in a comparable manner to that of experienced therapists.

\section{Method}

\section{Study Design}

Data were collected in Berlin as part of a project conducted in the Department of Psychotherapy and Somatopsychology at Humboldt University of Berlin, in collaboration with the Department of Medical Psychology of University Medical Centre Hamburg-Eppendorf, from October 2013 until March 2014. The project was funded by the European Integration Fund (EIF) and ethical approval for the study was given by the Ethical Review Board of the Department of Psychology at Humboldt.

The conceptual underpinnings of the training programme evaluated in this study were based on the Guidelines for Inter-/transcultural Competence Training of Psychotherapists, which were developed during a previous project by our workgroup (von Lersner et al., 2016).

Data were acquired before (pre-), immediately after (post-) and three months after (follow-up) the training programme.

Participants were recruited through mailing lists of educational institutions for clinical psychologists and psychological associations in Berlin. We included five institutes for cognitive behavioural therapy as well as five for psychoanalysis and depth psychology. 
The main inclusion criterion for participation in the training programme was having had professional training in psychotherapy for adult clients. Therapists who were still in training had to have passed on to the practical stage and be treating clients under supervision. A control group that did not participate in the programme was polled online during the pre-measurement phase. Participation in both groups was voluntary, based on informed consent and without any incentives or remuneration. Unfortunately, as we recruited participants through mailing lists, we were not able to track a reliable response rate.

Figure 1 illustrates the flow of participants in the course of the study.

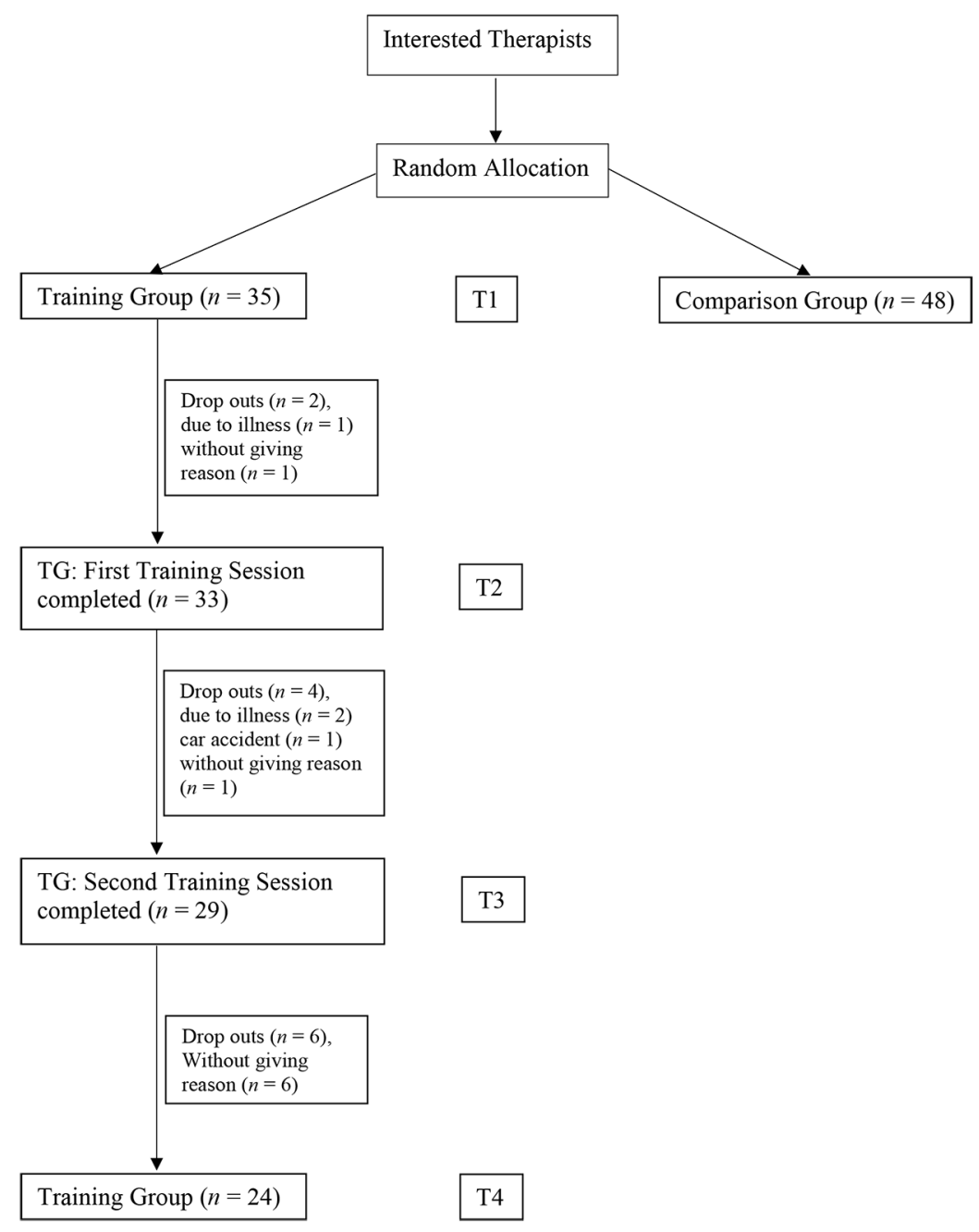

Figure 1. Flow of participants. 


\section{Implementation of the Training Programme}

Before implementation of the training programme, a pilot training session was carried out with psychology students. Subsequent adjustments were made for the final training manual which would guide the training programme analysed here.

The intervention took place in Berlin and consisted of two consecutive days of training-16.5 hours in total. Three weeks later, an additional, one-day refresher session was carried out consisting of six units of 45 minutes each ( 4.5 hours total). Experienced therapists and therapists in training received separate training circuits, which were run by experienced intercultural trainers from the project team.

\section{Programme Content}

The learning objectives defined in the aforementioned Guidelines for Intercultural Competence (von Lersner et al., 2016) formed the basis of the content of the training programme. As recommended by Hager, Patry, and Brezing (2000), an outline of the training programme was discussed by an expert panel (consisting of the project team and four experienced intercultural trainers from the University Medical Centre Hamburg-Eppendorf and the Transcultural Centre in Stockholm), and subsequent adaptations incorporated into the final version by the project team. Table 1 provides a brief overview of the different aspects of intercultural competence covered by individual modules of the programme. In terms of the didactic methods employed, input lectures, self-reflective exercises, critical incident technique, plenary discussions and role play were included.

Table 1

Overview of the Training Modules

\begin{tabular}{|c|c|}
\hline Module & Content \\
\hline Culture & $\begin{array}{l}\text { e.g. definition of culture in the personal and therapeutic context, } \\
\text { transcultural competence, ethnocentrism, diversity }\end{array}$ \\
\hline Migration & $\begin{array}{l}\text { e.g. information on facts and figures on migration pathways, } \\
\text { stressors \& resources, political framework }\end{array}$ \\
\hline $\begin{array}{l}\text { Experiencing cultural foreignness in } \\
\text { everyday life }\end{array}$ & $\begin{array}{l}\text { e.g. personal experiences of cultural foreignness, stereotypes \& } \\
\text { prejudices, individual norms and values, discrimination }\end{array}$ \\
\hline $\begin{array}{l}\text { Working with cultural brokers and } \\
\text { interpreters }\end{array}$ & $\begin{array}{l}\text { e.g. the meaning of language in the therapeutic process, language } \\
\text { barriers, rules for the inclusion of interpreters in the therapeutic } \\
\text { setting }\end{array}$ \\
\hline
\end{tabular}




\begin{tabular}{|c|c|}
\hline Module & Content \\
\hline $\begin{array}{l}\text { Psychometric testing \& classification of } \\
\text { mental disorders }\end{array}$ & $\begin{array}{l}\text { e.g. opportunities and limitations of culture-sensitive testing, } \\
\text { epidemiology of mental disorders in different cultural settings, } \\
\text { culture-specific symptom presentation, cultural concepts of } \\
\text { distress in DSM-5 }\end{array}$ \\
\hline Exploration and anamnesis & $\begin{array}{l}\text { e.g. Cultural Formulation Interview, culture-specific explanatory } \\
\text { models of mental illness }\end{array}$ \\
\hline $\begin{array}{l}\text { Experiencing cultural foreignness in the } \\
\text { clinical setting }\end{array}$ & e.g. critical incidents \\
\hline $\begin{array}{l}\text { Booster Session } \\
\text { (three weeks later) }\end{array}$ & $\begin{array}{l}\text { e.g. supervision, clarification of outstanding questions, } \\
\text { identification of 'cultural pitfalls' (Auernheimer, 2002) on the } \\
\text { basis of own examples }\end{array}$ \\
\hline
\end{tabular}

\section{Measures}

\section{Sociodemographic Data}

Basic variables regarding the sociodemographic background of participants-gender, age, migration background, therapeutic approach and first language-were recorded. Further, information regarding prior experience of intercultural competence training as well as level of personal interest in the topic was collected.

\section{Personality Traits}

In order to assess the influence of the personality trait of openness, the survey also included the short version of the Big Five Inventory (BFI-10; Rammstedt \& John, 2005), which measures the Big Five Personality dimensions using just two items for each of them. Rammstedt (2007) reports satisfactory values for the test's quality criteria, and the items on openness exhibit moderate values for retest reliability $\left(r_{t t}=.62\right)$.

\section{Intercultural Competence}

The intercultural competence of participants was measured via the widely used four-dimension Multicultural Counseling Inventory (MCI; Sodowsky, Taffe, Gutkin, \& Wise, 1994). The MCI is based on the three dimensions of intercultural competence formulated by Sue and Sue (2012)-knowledge, awareness and skills-but also seeks to capture an additional, fourth dimension: the multicultural therapeutic relationship. This four-factor structure allows single scores to be calculated for each of the dimensions as well as an overall sum score for intercultural competence. The 40 items of the MCI comprise statements about counselling and therapy in intercultural settings. Participants are asked to respond on a five-point Likert scale, ranging from 1 = 'very inaccurate' to 5 = 'very accurate'. For the present study, the MCI was translated into German via the back-translation 
method (Brislin, 1970) and adapted linguistically from counselling to therapeutic settings. The MCI meets the requirements for questionnaires in terms of reliability $(.71 \leq \alpha \leq .90)$ as well as validity. In our sample, it had a Cronbach's alpha $\alpha=.88$ at $\mathrm{T}_{1}, \alpha=.88$ at $\mathrm{T}_{3}$ and $\alpha=.89$ at $\mathrm{T}_{4}$, which represent good values. Cronbach's alphas for the subscales were as follows: $\alpha_{\text {relationship }}=.68, \alpha_{\text {awareness }}=.78, \alpha_{\text {knowledge }}=.85$ and $\alpha_{\text {skills }}=.79$ at $\mathrm{T}_{1 ;} \alpha_{\text {relationship }}$ $=.63, \alpha_{\text {awareness }}=.80, \alpha_{\text {knowledge }}=.80$ and $\alpha_{\text {skills }}=.76$ at $\mathrm{T}_{3}$ and, $\alpha_{\text {relationship }}=.67, \alpha_{\text {awareness }}$ $=.82, \alpha_{\text {knowledge }}=.79$ and $\alpha_{\text {skills }}=.84$ at $\mathrm{T}_{4}$.

\section{Therapeutic Relationship}

In addition to the multicultural therapeutic relationship dimension of the MCI, a closer examination of the therapeutic relationship was achieved using the German version of the Scale to Assess the Therapeutic Relationship in Community Mental Health Care, Clinician Version (STAR-C; McGuire-Snieckus, McCabe, Catty, Hansson, \& Priebe, 2007). The self-report STAR-C questionnaire consists of 12 items that seek to evaluate the quality of the therapeutic relationship from the perspective of the therapist. It consists of three factors: 1) positive collaboration, 2) positive clinician input and 3) emotional difficulties. Participants rated the therapeutic relationship separately for their clients with and without a migration background. Retest reliability of the German version is $r=.54$, which is satisfactory (Gairing, Jäger, Ketteler, Rössler, \& Theodoridou, 2011). In our sample, Cronbach's alphas were as follows: $\alpha_{\text {STAR_German clients }}=.67$ and $\alpha_{\text {STAR_Immigrant clients }}$ $=.42$ at $\mathrm{T}_{1} ; \alpha_{\text {STAR_German clients }}=.64$ and $\alpha_{\text {STAR_Immigrant clients }}=.45$ at $\mathrm{T}_{3} ;$ and, $\alpha_{\text {STAR_German }}$ clients $=.64$ and $\alpha_{\text {STAR_Immigrant clients }}=.62$ at $\mathrm{T}_{4}$ which reflect moderate to poor values.

Figure 2 gives an overview of the research design and the measures used at different sections of the study. Evaluation at $\mathrm{T}_{1}$ and $\mathrm{T}_{4}$ was carried out online, whereas at $\mathrm{T}_{2}$ and $\mathrm{T}_{3}$ data was collected in a paper and pencil format.

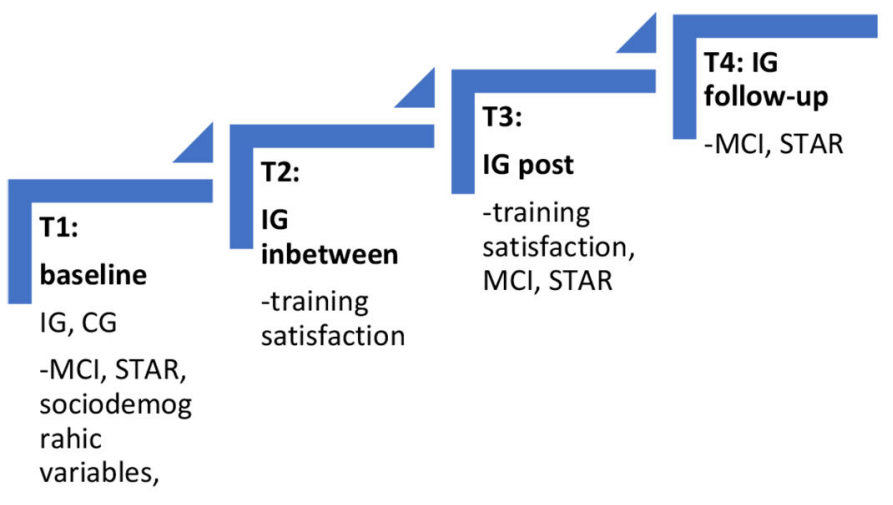

covariables

Figure 2. Overview of the research design.

Note. $\mathrm{IG}=$ Intervention group. $\mathrm{CG}=$ Control group. 


\section{Statistical Analysis}

Data analysis was carried out using SPSS (Version 22.0). In order to compare characteristics of the intervention and the control group, between-subject descriptive variables were compared using independent-sample $t$-tests or Wilcoxon rank-sum tests for continuous data and chi-square analysis for categorical data. Within-subject descriptive variables were compared using either paired-sample $t$-tests or Wilcoxon signed-rank tests, depending on data level.

Internal consistency of the STAR-C, BFI and MCI variables and therefore the reliability of the measures used in this study was examined by calculating Cronbach's alpha for the pre-, post- and follow-up total scores.

In order to control for possible selection bias for the variables of prior intercultural competence (MCI pre) and prior knowledge, as well as proportion of immigrant clients, personal interest and openness, univariate ANOVAs were carried out. Post-hoc tests using Bonferroni correction allowed us to localise the effects.

Further, to calculate the influence of the training programme on intercultural competence the Friedman test was used. For localisation of the effects over time, Wilcoxon signed-rank tests were applied to non-parametric data and $t$-tests to parametric data. Meanwhile, interaction between time and status of participants (trainee vs. experienced therapist) was examined in a MANOVA with repeated measures.

Throughout the whole study, effect size is reported as Cohen's $d, r$ and $\omega$. An alpha of .05 was used to define statistical significance in all analyses, and power analysis was carried out using G*Power (Faul, Erdfelder, Lang, \& Buchner, 2007).

\section{Results}

\section{Sample Characteristics}

At T1 the intervention group consisted of 35 participants. Table 2 shows demographic characteristics of this group in comparison to the control group. Statistical analysis showed significant differences between the groups in terms of age and therapy method with the control group being younger and containing a larger percentage of CBT therapists. 
Table 2

Demographic Variables of Training Group and Control Group at T1

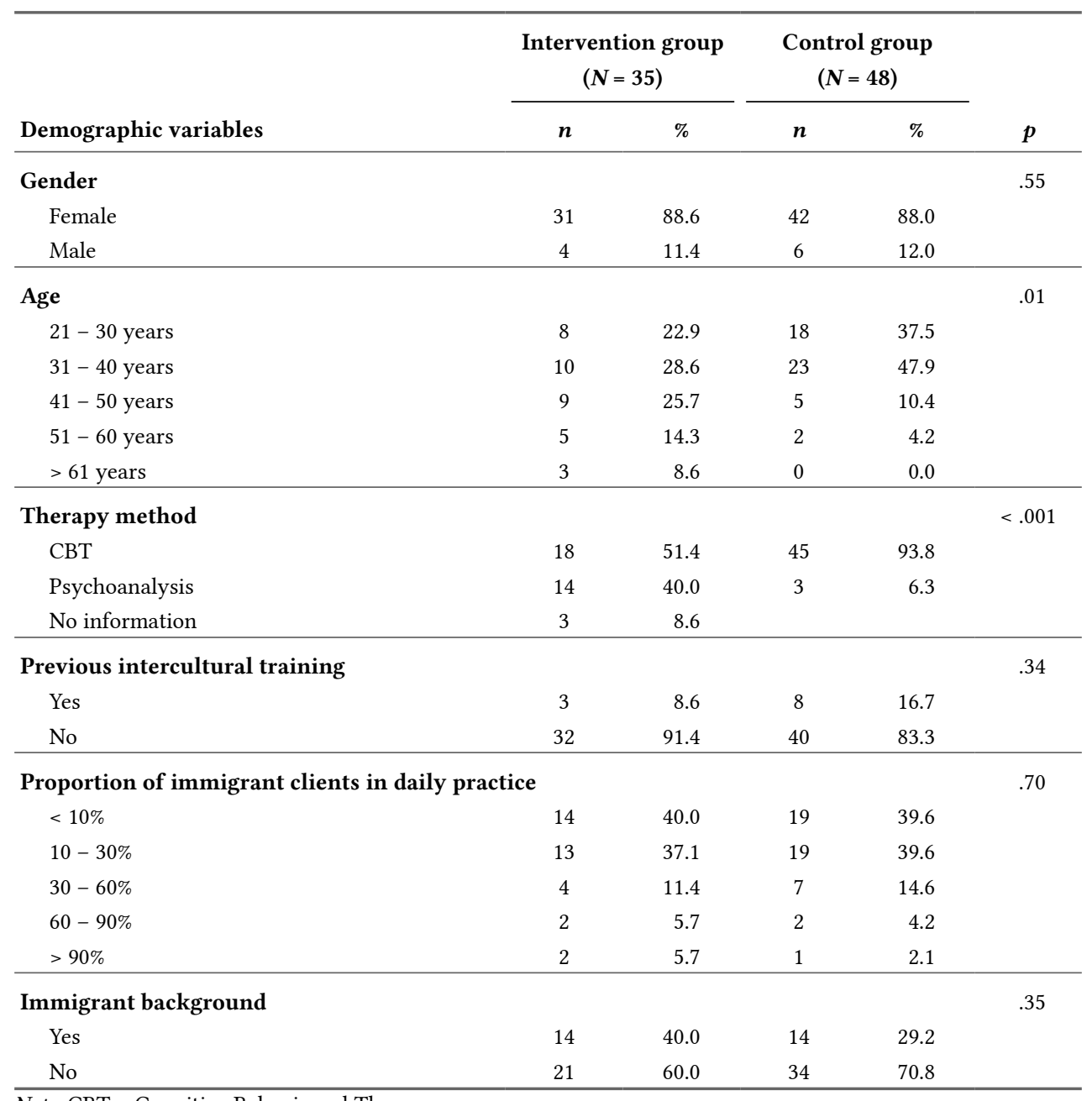

Note. CBT $=$ Cognitive Behavioural Therapy.

${ }^{*} p \leq .05 .{ }^{* *} p \leq .01$.

The whole training programme and evaluation was completed by $N=24$ participants, $33 \%$ of whom were between 31 and 40 years old and $82 \%$ female; $58 \%$ were trained (or still in training) in CBT; and 13\% had previously participated in intercultural training programmes lasting on average six hours. Regarding prior experience of immigrant clients, a quarter of participants reported that immigrants made up about $30 \%$ of their clientele, whereas the remaining participants treated significantly fewer. Furthermore, 38\% of 
participants had a migration background themselves, meaning that either they themselves or one of their parents had immigrated to Germany (Federal Statistics Office, 2016).

\section{Prerequisites and Verification of Selection Bias}

The verification of differences between the intervention and control groups before training revealed no significant differences in terms of intercultural competence before training (MCI-Pre), $t(68.53)=-1.35, p=.183$, proportion of immigrant clients, $H=2.739$, $p=.434, \chi^{2}(4)=3.01, p=.556$, or openness $(W=726.5, p=.361)$. However, there were significant differences between the intervention group and control group regarding interest $(W=1509.5, p<.001)$ and prior intercultural knowledge $(W=261, p<.001)$. Within groups, i.e. between trainees and therapists, no significant differences in mean values were detected.

\section{Intercultural Competence}

Table 3 shows the development of intercultural competence over time by presenting the results of the four subscales as well as the total score for the MCI.

Table 3

Intercultural Competence on the MCI Over the Course of the Training

\begin{tabular}{|c|c|c|c|c|c|c|c|c|c|}
\hline \multirow[b]{2}{*}{ Sub-scale of the MCI } & \multicolumn{2}{|c|}{$\begin{array}{l}\text { Pre } \\
\text { T1 }\end{array}$} & \multicolumn{2}{|c|}{$\begin{array}{l}\text { Post } \\
\text { T3 }\end{array}$} & \multicolumn{2}{|c|}{$\begin{array}{c}\text { Follow-up } \\
\text { T4 }\end{array}$} & \multirow{2}{*}{$\begin{array}{c}\text { Pre- } \\
\text { Post } \\
d\end{array}$} & \multirow{2}{*}{$\begin{array}{c}\text { Pre- } \\
\text { Follow-up } \\
d\end{array}$} & \multirow{2}{*}{$\begin{array}{c}\text { Post- } \\
\text { Follow-up } \\
\text { d }\end{array}$} \\
\hline & $M$ & $S D$ & $M$ & $S D$ & $M$ & $S D$ & & & \\
\hline Skills & 3.80 & 0.51 & 3.75 & 0.51 & 3.93 & 0.40 & -.10 & $.28^{* *}$ & $.39^{*}$ \\
\hline Awareness & 3.09 & 0.86 & 3.32 & 0.67 & 3.33 & 0.74 & $.35^{*}$ & $.30^{*}$ & .01 \\
\hline Therapeutic Relationship & 3.39 & 0.46 & 3.38 & 0.47 & 3.54 & 0.31 & -.02 & .38 & $.40^{* *}$ \\
\hline Knowledge & 3.22 & 0.58 & 3.51 & 0.50 & 3.64 & 0.48 & $.54^{*}$ & $.79^{* *}$ & .27 \\
\hline Total Score & 3.41 & 0.46 & 3.52 & 0.30 & 3.62 & 0.33 & $.28^{*}$ & $.53^{* \star}$ & $.32^{*}$ \\
\hline
\end{tabular}

The data indicate a significant effect of time as a variable across both groups (experienced therapists and therapists in training, $\left.\chi^{2}(2, N=24)=17.70, p<.01, \omega=.86\right)$. The Wilcoxon signed-rank test revealed significant changes between all three measurement times (Pre to Post: $z=-3.29, p<.01, \varphi=.67,1-\beta=.43$; Post- to Follow-up: $z=-2.29, p$ $<.001, \varphi=.47,1-\beta=.44$; Pre to Follow-up: $z=-4.00, p<.01, \varphi=.82,1-\beta=.79)$. Significant changes on the dimensions of awareness and knowledge occurred immediately after training, whereas values for skills and therapeutic relationship increased significantly at follow-up. The status of trainees had no influence on training outcomes, meaning that 
experienced therapists and therapists in training benefitted from the training programme to the same degree, $F(2,44)=2.23, p=.12, \eta^{2}=.09$.

The therapeutic relationship, as seen from the perspective of the therapist, was investigated in further detail using STAR-C. Thus, participants were asked at $\mathrm{T}_{1}, \mathrm{~T}_{3}$ and $\mathrm{T}_{4}$ to rate their therapeutic relationships with their non-immigrant and immigrant clients, separately.

Statistical analysis revealed significant improvement in the therapeutic relationship with non-immigrant clients from $\mathrm{T}_{1}$ to $\mathrm{T}_{3}, t(28)=-1.73, p=.047$, and a clear trend toward significant change from $\mathrm{T}_{1}$ to $\mathrm{T}_{4}, t(24)=-1.47, p=.076$, and $\mathrm{T}_{3}$ to $\mathrm{T}_{4}, t(22)=-0.43, p=.334$. Yet, in contrast, no significant changes were observed with immigrant clients, $\mathrm{T}_{1}$ to $\mathrm{T}_{3}$ : $t(28)=-0.58, p=.284, \mathrm{~T}_{1}$ to $\mathrm{T}_{4}: t(24)=-1.40, p=.086$, and $\mathrm{T}_{3}$ to $\mathrm{T}_{4} t(22)=-0.82, p=.210$ (see Table 4).

Table 4

Therapeutic Relationship Over the Course of the Training Measured With STAR-C

\begin{tabular}{|c|c|c|c|c|c|c|c|c|c|}
\hline \multirow{2}{*}{$\begin{array}{l}\text { Therapeutic } \\
\text { Relationship }\end{array}$} & \multicolumn{2}{|c|}{$\begin{array}{l}\text { Pre } \\
\text { T1 }\end{array}$} & \multicolumn{2}{|c|}{$\begin{array}{c}\text { Post } \\
\text { T3 }\end{array}$} & \multicolumn{2}{|c|}{$\begin{array}{c}\text { Follow-up } \\
\text { T4 } \\
\end{array}$} & \multirow{2}{*}{$\begin{array}{c}\text { Pre- } \\
\text { Post } \\
d\end{array}$} & \multirow{2}{*}{$\begin{array}{c}\text { Pre- } \\
\text { Follow up } \\
\qquad\end{array}$} & \multirow{2}{*}{$\begin{array}{c}\text { Post- } \\
\text { Follow up } \\
\text { d }\end{array}$} \\
\hline & $M$ & $S D$ & $M$ & $S D$ & $M$ & $S D$ & & & \\
\hline Non-immigrant clients & 3.40 & .39 & 3.47 & .26 & 3.52 & .25 & .21 & .37 & .20 \\
\hline Immigrant clients & 3.35 & .30 & 3.37 & .25 & 3.46 & .30 & .07 & .37 & .33 \\
\hline
\end{tabular}

\section{Discussion}

In this pilot study we evaluated an intercultural training programme for psychotherapists, focusing particularly on changes over time in the intercultural competence of participants as well as the therapeutic relationship from the perspective of the therapist. In order to control for a possible selection bias among participants a control group was included at $\mathrm{T}_{1}$ and we measured variables such as interest in intercultural issues prior to training and prior knowledge and experience of immigrant clients, as well as work experience.

International evaluation studies point to a general effectiveness of intercultural competency training (Benish et al., 2011; Betancourt \& Green, 2010; Kalinoski et al., 2013; Kulik \& Roberson, 2008), and our first evaluation of one such training programme in Germany follows these studies in its overall assessment. Even though intercultural competence training programmes for therapists existed prior to our study, no systematic evaluation had been carried out with this target group.

Applying our approach, intercultural competence as measured with the MCI increased significantly. This was the case for the total score as well as the separate scores for each of the four dimensions of the inventory. 
Our training programme also had an immediate effect on the dimensions of awareness and knowledge, as described by Beach et al. (2005). In the domain of awareness, this implies, for example, reflecting on one's own prejudices and examining stereotypes so as to develop better awareness of and openness to cultural diversity, and learning about the interplay between one's own attitudes towards immigrant clients and the therapeutic process. Meanwhile, increases in the domain of knowledge imply being better informed about the diversity of cultural groups, differences in cultural concepts of distress and styles of communication, and the use of cultural brokers in therapy. It may also include greater understanding of the socio-political contexts in which intercultural therapy takes place. These are areas of improved intercultural competence that can be absorbed rather quickly and immediately.

In contrast, effects on the dimensions of skills and the therapeutic relationship, again as measured by the MCI, only became evident at the three-month follow-up. As already presumed by Kulik and Roberson (2008), it seems that such changes only become prevalent with direct interaction with clients; thus, these aspects can only be reliably evaluated after participants have returned to work rather than immediately after participation in the programme.

Campinha-Bacote (2002) and Guzder and Rousseau (2013) describe the development of intercultural competence as an ongoing process in which trainees are constantly challenged to be aware of and question their own cultural imprints, thereby becoming able to take their clients' different cultural backgrounds into account in therapy. But critical analysis of one's own values and norms, and the acquiring of new skills, are comprehensive and long-term developmental processes that do not happen overnight. Interestingly, though, the results of the present study indicate that this process can be successfully initiated through training programmes like the one presented here.

Our comparison of the study group and the control group revealed that groups did not differ in terms of intercultural competence before training, proportion of immigrant clients or openness but did differ in terms of interest and prior intercultural knowledge. We cautiously take this to assume that selection bias can thus be excluded, and that by and large training outcomes can be attributed solely to the training programme. At the same time, we have to consider that the sample of this pilot study was too small to carry out reliable regression analyses and to safely rule out a selection bias. This shortcoming could be addressed in future research by larger samples and the inclusion of a control group across all times of measurement.

One reason for the positive outcome of the programme could be the teaching methods used. According to Kalinoski et al. (2013), emotion-focused techniques such as critical incident technique, self-reflective units or active sessions can be more effective in intercultural training than simply imparting theoretical knowledge. The training programme that we developed, implemented and evaluated here includes a high proportion of emotion-activating methods throughout. In each module, a brief introduction is followed by 
exercises in which participants can try out a new approach, discuss case vignettes or reflect upon their own perspective. Kalinoski et al. (2013) also demonstrated that training sessions distributed over two or more points in time tend to be more effective compared with longer, one-off interventions. As our training programme consisted of core or primary training sessions spread over two days and an additional refresher session three weeks later, this could also account for the positive outcomes observed. Besides effective training methods and structure, an additional success factor could be the greater specificity of the target group we selected compared with those participating in previous training programmes, given that the programme was specifically both developed for and evaluated by psychotherapists.

As we have mentioned, there was no significant difference in programme outcomes between experienced therapists and therapists in training, suggesting that the programme is appropriate for all therapists regardless of level of experience. This is important for the practical applicability of the programme, as one can either use one programme for all therapists or develop separate curricula for participants of different experience levels. The data suggest that the level of therapeutic experience is not important and thus that the training programme could be applied in postgraduate training as well as in trainings at a later point in therapists' careers. This finding is in line with the Guidelines for Intercultural Training (Kirmayer et al., 2012, von Lersner et al., 2016), which are based on the same assumption and define quality criteria for intercultural training across all groups of therapists.

\section{Therapeutic Relationship}

To assess the impact of the programme on the therapeutic process, we examined the perceived therapeutic relationship from the perspective of the participants over the course of the training. However, our study generated unexpected findings. Overall, from the perspective of the therapist, it seems that the therapeutic relationship benefits significantly from additional training. This was evident from the STAR-C post measurement as well as the relevant sub-domain of the MCI at follow-up. Yet, when participants were asked to rate the therapeutic relationship for non-immigrant and immigrant clients separately, significant improvement was observable for non-immigrant clients only. At first sight this would appear to be an unexpected outcome, given that the training programme focused on intercultural issues with the intention that immigrants should particularly benefit from it.

Two possible reasons for this counter-intuitive effect are considered here. First, if we recall the definition of culture on which our training programme is based, this effect should not be so surprising. According to Geertz (1973), culture consists of a set of values and norms shared by a group of people independently of their regional origin, a perspective that is also linked to the concept of diversity introduced earlier in this paper. Thus, during the training sessions regional origin and migration status were only two of nu- 
merous dimensions associated with culture and diversity. The results found here would seem to confirm this perspective: the quality of the therapeutic relationship improved for the majority of clients irrespective of their immigrant status, suggesting that factors such as openness, greater sensitivity towards clients' socio-cultural background and better awareness of one's own cultural norms and expectations can be beneficial to all clients.

Secondly, our sample size was rather small at $\mathrm{T}_{1}$ and had decreased further by the time of the follow-up, with the remaining therapists treating a rather small number of immigrants-at or below $30 \%$ of their clients-in their daily practice. Thus, it may be due to the small size of this reference group that improvements in the therapeutic relationship between these immigrant clients and their therapists were simply not readily apparent. At the same time, our statistical results clearly point to a positive trend regarding the effect of the training on the therapeutic relationship. This appears to have been confirmed when we used the MCI-on which only the therapeutic relationship with immigrant clients was rated-which reported significant improvement. Nonetheless, we feel that, as a consequence of this pilot study, the evaluation should be repeated with a larger sample and more reliable measures in the future. Internal consistencies of STAR-C scales were unsatisfactory and might have had a negative effect on the outcome values. Consideration should also be given to modifying the training programme's units on migrationrelated issues, so as to increase the likelihood that the therapeutic relationship with immigrant clients benefits in the same way as that with non-immigrant clients did. Furthermore, in order to achieve robust positive training outcomes, Guzder and Rousseau (2013) recommended ongoing supervision following training. This could support participants in terms of strengthening their newly acquired skills and dealing with any uncertainties arising in the process, both of which may have a long-term, positive impact on the therapeutic relationship.

\section{Limitations and Implications}

In addition to its positive outcomes, the study also has a number of limitations. Because of the small sample size, some statistical trends may have been imperceptible that may have been significant with a larger dataset. Also, effect sizes were relatively small. Another result of the small sample size is that our findings cannot be confidently generalised to all therapists. Thus, further studies with larger sample sizes are urgently needed to underpin the effects found in this study. In the meantime, in view of the lack of studies in this field internationally, as well as in Germany, our pilot data provide important information and empirical insights.

The statistical values of the STAR-C were also unsatisfactory, potentially reducing the explanatory power of our findings on the therapeutic relationship. This limitation should be addressed in future research. Nevertheless, significant effects of the programme were established in spite of the poor statistical values of the STAR-C and the small sample size. 
Hence, necessary further studies with larger samples are currently being carried out by our team.

In line with Kulik and Roberson (2008), we believe that in future research it would be interesting to evaluate the impact of single training units or specific interventions. In addition, shorter versions of the programme could be evaluated to increase the applicability of the approach in time-strapped clinical settings. Turning to methodological issues, we feel that in the intercultural therapy context it is difficult using questionnaires to measure awareness or attitudes as they may manifest instead at the behavioural level. Hence, it might be useful to examine video recordings of therapy sessions-with real or simulated clients, before and after training intervention-to examine the behavioural level more directly. Other indicators of behavioural change resulting from the training might be client satisfaction with treatment, duration of treatment, percentage of immigrant clients and immigrant-client drop-out rates. It would also appear to be necessary to evaluate client perspectives on the therapeutic relationship, especially those of immigrants.

\section{Conclusions}

We conclude from this first evaluation of our intercultural training programme that it offers an effective intervention in terms of enabling psychotherapists to be more culturally sensitive towards clients from migration backgrounds. Such effects were demonstrable to our satisfaction, even with our small sample. We suggest that this training programmewhich is actually a rather brief intervention-could lead to significant improvements in therapeutic practice in crucial ways.

Funding: The authors received no financial support for the research, authorship, and/or publication of this article.

Competing Interests: The authors declare no conflicts of interest.

Acknowledgments: The authors have no support to report.

\section{References}

Aggarwal, N. K., Cedeño, K., Guarnaccia, P., Kleinman, A., \& Lewis-Fernández, R. (2016). The meanings of cultural competence in mental health: An exploratory focus group study with patients, clinicians, and administrators. SpringerPlus, 5(1), Article 384.

https://doi.org/10.1186/s40064-016-2037-4

American Psychological Association. (2003). Guidelines on multicultural education, training, research, practice, and organizational change for psychologists. American Psychologist, 58(5), 377-402. https://doi.org/10.1037/0003-066X.58.5.377 
American Psychological Association. (2008). Report of the task force on the implementation of the multicultural guidelines. Washington, DC, USA: Author. Retrieved from http://www.apa.org/pi/

Auernheimer, G. (2002). Interkulturelle Kompetenz und Professionalität (Interkulturelle Studien, 13). Opladen, Germany: Leske und Budrich.

Beach, M. C., Price, E. G., Gary, T. L., Robinson, K. A., Gozu, A., Palacio, A., . . Powe, N. R. (2005). Cultural competency: A systematic review of health care provider educational interventions. Medical Care, 43(4), 356-373. https://doi.org/10.1097/01.mlr.0000156861.58905.96

Benish, S. G., Quintana, S., \& Wampold, B. E. (2011). Culturally adapted psychotherapy and the legitimacy of myth: A direct comparison meta-analysis. fournal of Counselling Psychology, 58(3), 279-289. https://doi.org/10.1037/a0023626

Bennegadi, R. (2009). Cultural competence and training in mental health practice in Europe: Strategies to implement competence and empower practitioners. Paris, France: Minkowska Centre.

Betancourt, J. R., \& Green, A. R. (2010). Linking cultural competence training to improved health outcomes: Perspectives from the field. Academic Medicine, 85(4), 583-585. https://doi.org/10.1097/ACM.0b013e3181d2b2f3

Brislin, R. W. (1970). Back-translation for cross-cultural research. Journal of Cross-Cultural Psychology, 1(3), 185-216. https://doi.org/10.1177/135910457000100301

Campinha-Bacote, J. (2002). The process of cultural competence in the delivery of healthcare services: A model of care. Journal of Transcultural Nursing, 13, 181-184. https://doi.org/10.1177/10459602013003003

Chen, J., \& Rizzo, J. (2010). Racial and ethnic disparities in use of psychotherapy: Evidence from U.S. National Survey data. Psychiatric Services, 61(4), 364-372. https://doi.org/10.1176/ps.2010.61.4.364

Chipps, J. A., Simpson, B., \& Brysiewicz, P. (2008). The effectiveness of cultural-competence training for health professionals in community-based rehabilitation: A systematic review of literature. Worldviews on Evidence-Based Nursing, 5, 85-94.

https://doi.org/10.1111/j.1741-6787.2008.00117.x

Claassen, D., Ascoli, M., Berhe, T., \& Priebe, S. (2005). Research on mental disorders and their care in immigrant populations: A review of publications from Germany, Italy and the UK. European Psychiatry, 20(8), 540-549. https://doi.org/10.1016/j.eurpsy.2005.02.010

de Haan, A. M., Boon, A. E., de Jong, J. T. V. M., \& Vermeiren, R. R. J. M. (2018). A review of mental health treatment dropout by ethnic minority youth. Transcultural Psychiatry, 55(1), 3-30. https://doi.org/10.1177/1363461517731702

Delgado, D. A., Ness, S., Ferguson, K., Engstrom, P. L., Gannon, T. M., \& Gillett, C. (2013). Cultural competence training for clinical staff: Measuring the effect of a one-hour class on cultural competence. Fournal of Transcultural Nursing, 24, 204-213. https://doi.org/10.1177/1043659612472059

Faul, F., Erdfelder, E., Lang, A. G., \& Buchner, A. (2007). G* Power 3: A flexible statistical power analysis program for the social, behavioral, and biomedical sciences. Behavior Research Methods, 39(2), 175-191. https://doi.org/10.3758/BF03193146 
Federal Statistics Office. (2016). Zahlen und Fakten: Migration und Integration. Retrieved from https://www.destatis.de/DE/ZahlenFakten/GesellschaftStaat/Bevoelkerung /

MigrationIntegration/MigrationIntegration.html

Gairing, S. K., Jäger, M., Ketteler, D., Rössler, W., \& Theodoridou, A. (2011). "Scale to assess therapeutic relationships, STAR": Evaluation der deutschen Skalenversion zur Beurteilung der therapeutischen Beziehung. Psychiatrische Praxis, 38(4), 178-184.

https://doi.org/10.1055/s-0030-1265979

Geertz, C. (1973). Dichte Beschreibung. Beiträge zum Verstehen kultureller Systeme. Frankfurt am Main, Germany: Suhrkamp.

Guzder, J., \& Rousseau, C. (2013). A diversity of voices: The McGill 'Working with Culture' seminars. Culture, Medicine, and Psychiatry, 37(2), 347-364.

https://doi.org/10.1007/s11013-013-9316-0

Hager, W., Patry, J., \& Brezing, H. (Eds.). (2000). Evaluation psychologischer Interventionsmassnahmen: Standards und Kriterien: Ein Handbuch. Göttingen, Germany: Hogrefe.

Kalinoski, Z. T., Steele-Johnson, D., Peyton, E. J., Leas, K. A., Steinke, J., \& Bowling, N. A. (2013). A meta-analytic evaluation of diversity training outcomes. fournal of Organizational Behavior, 34(8), 1076-1104. https://doi.org/10.1002/job.1839

Khanna, S. K., Cheyney, M., \& Engle, M. (2009). Cultural competency in health care: Evaluating the outcomes of a cultural competency training among health care professionals. Fournal of the National Medical Association, 101(9), 886-892. https://doi.org/10.1016/S0027-9684(15)31035-X

Kirmayer, L. J. (2012). Rethinking cultural competence. Transcultural Psychiatry, 49(2), 149-164. https://doi.org/10.1177/1363461512444673

Kirmayer, L. J., Fung, K., Rousseau, C., Tat Lo, H., Menzies, P., Guzder, J., ... McKenzie, K. (2012). Guidelines for training in cultural psychiatry. Ottawa, Canada: Canadian Psychiatric Association.

Kirmayer, L. J., Narasiah, L., Munoz, M., Rashid, M., Ryder, A. G., Guzder, J., . . Pottier, K. (2011). Common mental health problems in immigrants and refugees: General approach in primary care. Canadian Medical Association fournal, 183(12), E959-E967.

https://doi.org/10.1503/cmaj.090292

Koch, E., Hartkamp, N., Siefen, R. G., \& Schouler-Ocak, M. (2008). Patienten mit Migrationshintergrund in stationär-psychiatrischen Einrichtungen. Nervenarzt, 79, 328-339. https://doi.org/10.1007/s00115-007-2393-y

Kulik, C. T., \& Roberson, L. (2008). Common goals and golden opportunities: Evaluations of diversity education in academic and organizational settings. Academy of Management Learning \& Education, 7(3), 309-331. https://doi.org/10.5465/amle.2008.34251670

Lie, D. A., Lee-Rey, E., Gomez, A., Bereknyei, S., \& Braddock, C. H. (2011). Does cultural competency training of health professionals improve patient outcomes? A systematic review and proposed algorithm for future research. fournal of General Internal Medicine, 26(3), 317-325. https://doi.org/10.1007/s11606-010-1529-0 
Lindert, J., Priebe, S., Penka, S., Napo, F., Schouler-Ocak, M., \& Heinz, A. (2008). Versorgung psychisch kranker Patienten mit Migrationshintergrund. Psychotherapie Psychosomatik Medizinische Psychologie, 58(03/04), 123-129. https://doi.org/10.1055/s-2008/1067360

Machleidt, W., Behrens, K., Ziegenbein, M., \& Calliess, I. T. (2007). Integration von Migranten in die psychiatrisch-psychotherapeutische Versorgung in Deutschland. Psychiatrische Praxis, 34(7), 325-331. https://doi.org/10.1055/s-2007-986192

McGuire-Snieckus, R., McCabe, R., Catty, J., Hansson, L., \& Priebe, S. (2007). A new scale to assess the therapeutic relationship in community mental health care: STAR. Psychological Medicine, 37(1), 85-95. https://doi.org/10.1017/S0033291706009299

Mösko, M. O., Baschin, K., Längst, G., \& von Lersner, U. (2012). Interkulturelle Trainings für die psychosoziale Versorgung. Psychotherapeut, 57(1), 15-21.

https://doi.org/10.1007/s00278-011-0878-4

Norcross, J. C. (2010). The therapeutic relationship. In B. L. Duncan, S. D. Miller, B. E. Wampold, \& M. A. Hubble (Eds.), The heart and soul of change: Delivering what works in therapy (pp. 113-141). https://doi.org/10.1037/12075-004

Price, E. G., Beach, M. C., Gary, T. L., Robinson, K. A., Gozu, A., Palacio, A., . . Cooper, L. A. (2005). A systematic review of the methodological rigor of studies evaluating cultural competence training of health professionals. Academic Medicine, 80, 578-586. https://doi.org/10.1097/00001888-200506000-00013

Rammstedt, B. (2007). The 10-Item Big Five Inventory (BFI-10): Norm values and investigation of sociodemographic effects based on a German population representative sample. European fournal of Psychological Assessment, 23, 193-201. https://doi.org/10.1027/1015-5759.23.3.193

Rammstedt, B., \& John, O. P. (2005). Kurzversion des Big Five Inventory (BFI-K). Diagnostica, 51(4), 195-206. https://doi.org/10.1026/0012-1924.51.4.195

Sodowsky, G. R., Taffe, R. C., Gutkin, T. B., \& Wise, S. L. (1994). Development of the Multicultural Counseling Inventory: A self-report measure of multicultural competencies. fournal of Counseling Psychology, 41(2), 137-148. https://doi.org/10.1037/0022-0167.41.2.137

Steinhäuser, T., Martin, L., von Lersner, U., \& Auckenthaler, A. (2014). Konzeptionen von "transkultureller Kompetenz" und ihre Relevanz für die psychiatrisch-psychotherapeutische Versorgung. Ergebnisse eines disziplinübergreifenden Literaturreviews. Psychotherapie Psychosomatik Medizinische Psychologie, 64(09/10), 345-353.

https://doi.org/10.1037/0022-0167.41.2.137

Sue, D. W., \& Sue, D. (1990). Counseling the culturally different: Theory \& practice (2nd ed.). New York, NY, USA: John Wiley.

Sue, D. W., \& Sue, D. (2012). Counseling the culturally diverse: Theory and practice (6th ed.). Hoboken, NJ, USA: John Wiley \& Sons.

Ta, T. M. T., Neuhaus, A. H., Burian, R., Schomerus, G., von Poser, A., Diefenbacher, A., . . Hahn, E. (2015). Inanspruchnahme ambulanter psychiatrischer Versorgung bei vietnamesischen Migranten der ersten Generation in Deutschland. Psychiatrische Praxis, 42(05), 267-273. https://doi.org/10.1055/s-0034-1370008 
van Keuk, E., Ghaderi, C., Joksimovic, L., \& David, M. (Eds.). (2011). Transkulturelle Kompetenz in klinischen und sozialen Arbeitsfeldern. Stuttgart, Germany: Kohlhammer.

von Lersner, U. (2015, January). Kultursensibel aber wie? Leitlinien für Trainings transkultureller Kompetenzen von Psychotherapeuten und Implikationen für die Praxis. Paper presented at the Berliner Psychiatrietage, Berlin, Germany.

von Lersner, U., Baschin, K., Wormeck, I., \& Mösko, M. (2016). Kultursensibel, aber wie? Leitlinien für Trainings inter-/ transkulturelle Kompetenzen in der Aus-, Fort- und Weiterbildung von Psychotherapeut_innen. Abschlusspublikation des Projektes. Retrieved from https://www.psychologie.hu-berlin.de/prof/the/Leitlinien/view

Wohlfart, E., Hodzic, S., \& Özbek, T. (2006). Transkulturelles Denken und transkulturelle Praxis in der Psychiatrie und Psychotherapie. In E. Wohlfart \& M. Zaumseil (Eds.), Transkulturelle Psychiatrie \& Interkulturelle Psychotherapie. Interdisziplinäre Theorie und Praxis (pp. 142-168). Heidelberg, Germany: Springer.

Yeo, S. (2004). Language barriers and access to care. Annual Review Nursery Research, 22(1), 59-73. https://doi.org/10.1891/0739-6686.22.1.59

\section{EACLIPT}

Clinical Psychology in Europe (CPE) is the official journal of the European Association of Clinical Psychology and Psychological Treatment (EACLIPT).

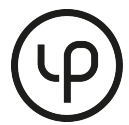

leibniz-psychology.org

PsychOpen GOLD is a publishing service by Leibniz Institute for Psychology Information (ZPID), Germany. 